\title{
Numerical study of breathers in a bent chain of oscillators with long-range interaction
}

\author{
J F R Archilla ${ }^{1}$, P L Christiansen ${ }^{2}$, S F Mingaleev ${ }^{3}$ and Yu B Gaididei ${ }^{3}$ \\ ${ }^{1}$ Department of Applied Physics I, University of Seville, Avda Reina Mercedes s/n, \\ 41012-Sevilla, Spain \\ ${ }^{2}$ Informatics and Mathematical Modelling, The Technical University of Denmark, DK-2800 \\ Lyngby, Denmark \\ 3 Bogolyubov Institute for Theoretical Physics, 03143 Kiev, Ukraine \\ E-mail: archilla@cica.es
}

Received 6 March 2001, in final form 12 June 2001

Published 10 August 2001

Online at stacks.iop.org/JPhysA/34/6363

\begin{abstract}
Most of the studies of breathers in networks of oscillators are limited to nextneighbour interaction. However, long-range interaction becomes critical when the geometry of the chain is taken into account, as the distance between oscillators and, therefore, the coupling, depends on the shape of the system. In this paper we analyse the existence and stability of breathers, i.e. localized oscillations in a simple model for a bent chain of oscillators with long-range interaction.
\end{abstract}

PACS numbers: 02.60.-x, 05.45.Xt, 05.50.+q, 63.20.Pw, 63.20.Ry, 63.50.+x, 87.10.+e

\section{Introduction}

During recent years a great deal of attention has been paid to localized oscillations in nonlinear discrete systems, which are usually known as discrete breathers, to differentiate them from the entity with the same name in continuous systems. In a basic mathematical paper by Mackay and Aubry [1], their existence and stability were established under rather general conditions. This mathematical proof, based on the limit with no coupling, has become one of the starting points for obtaining breathers, as 'exact' numerical solutions, up to machine precision [2-5]. A thorough review can be found in [6].

Many researchers have worked in the extension of the proofs to more general conditions, applications to specific fields and different systems and to obtain experimental evidence of their existence in real systems [7-12]. Work has been done on the interplay of nonlinearity and inhomogeneity or disorder [13-15]. 
The important and related point we address here is the influence of geometry, i.e. of the shape of the chain of oscillators, on the existence and stability of breathers, and therefore on localization of energy. Geometry is not relevant, as long as only nearest-neighbour interaction is taken into account, but with long-range interaction it becomes critical, as the distances between oscillators change and therefore the intensity of the coupling between them. The geometry cannot be neglected any longer.

Bending of Klein-Gordon models has been studied in several papers. In [16] the bending is introduced as an inhomogeneity in a model for DNA. Perturbative methods and numerical simulation are used. In [17], the bending is introduced as a change of the coupling constant, also for a DNA model. Numerical simulation is also used. Nonlinear Schrödinger equation (NLS) models with long-range interaction have been studied in straight chains $[18,19]$, a twodimensional system with dipole-dipole interaction [20] and bent chains [21,22]. Breathers with long-range interaction in the straight chain appear in [23,24]. However, the effect of curvature and long-range interaction has not yet been addressed in 'exact' breathers.

We choose a model as simple as possible to isolate the effect of the bending and the long-range interaction. We do not intend here the study of a specific physical model, but the effect of the curvature on the existence of localized oscillations that involve a few oscillators. Therefore, the long-range interaction we are interested in should have two characteristics: (a) it has to decay slowly enough so as the system can feel the curvature; (b) it has to decay fast enough so as the breather remain localized within a few units, i.e. the properties of the system are far away from the continuous limit. This is a very different situation with respect to the generic study of breathers with long-range interaction in the straight chain as is carefully done in [23,24]. Within these limitations, the exact form of the interaction is not qualitatively relevant. The main factors that affect the properties of breathers are the strength of the coupling and the curvature.

Our model consists of a Klein-Gordon model with long-range harmonic coupling, with cubic power decay: that is, a straightforward generalization of the Hamiltonian in [18], and as used for DNA in [19]. It is also a particular case of the generic studies in [23,24], described as the dipole-dipole case. The first two references deal with the NLS, and all four refer to straight chains. We use a soft on-site potential as it is the most common type used when biological systems are modelled. It is described in detail in the next section.

\section{The model}

As commented above, we use a Klein-Gordon model, augmented with long-range interaction, the Hamiltonian becoming

$$
H=\sum_{n=1}^{N}\left(\frac{1}{2} \dot{u}_{n}^{2}+\frac{1}{2} \omega_{0}^{2} u_{n}^{2}+s \Phi\left(u_{n}\right)+\frac{1}{4} \varepsilon \sum_{m=1}^{N} J_{n m}\left(u_{n}-u_{m}\right)^{2}\right) .
$$

The variables $u_{n}$ correspond to displacements perpendicular to a plane where the system is embedded. A sketch of the chain is shown in figure 1.

The on-site potential is given by

$$
V\left(u_{n}\right)=\frac{1}{2} \omega_{0}^{2} u_{n}^{2}+s \Phi\left(u_{n}\right)
$$

with $s \phi\left(u_{n}\right)$ being the non-harmonic part, and $s$ a parameter to tune the degree of nonlinearity. We have chosen a soft-symmetric potential, i.e. $s \Phi\left(u_{n}\right)=-\frac{1}{4} u_{n}^{4}$. The absolute value of $s$ is, in fact, irrelevant, as it can be chosen as $s=1$, with a suitable scaling of the variables $u_{n}$. Similarly, we can choose $\omega_{0}=1$, with a suitable change of the time scale. 


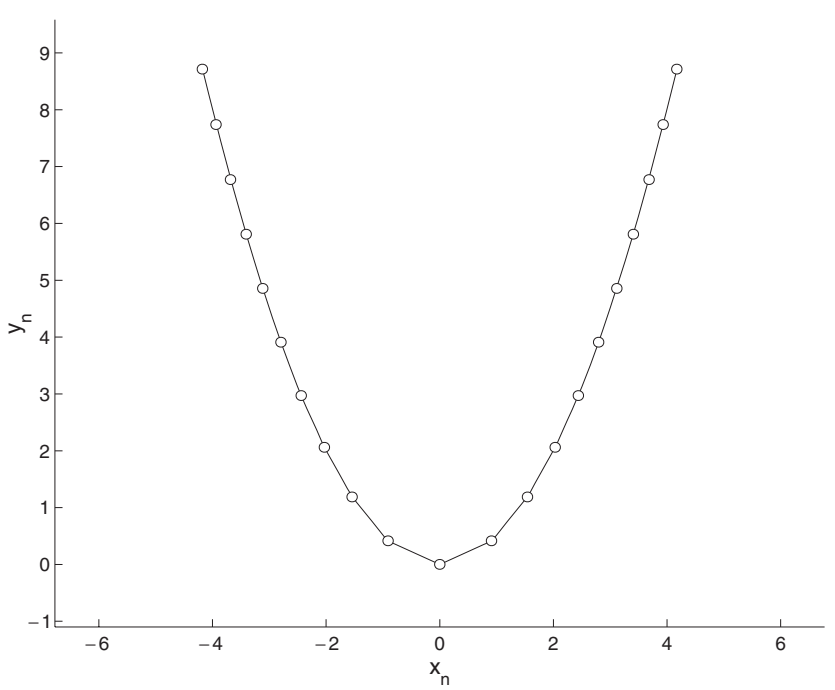

Figure 1. Sketch of the chain of oscillators with curvature $\kappa=1$.

The last term is the long-range interaction, $J_{n m}$ being the coupling constants (in time), with values, for $n \neq m, J_{n m}=1 /\left|\boldsymbol{r}_{n}-\boldsymbol{r}_{m}\right|^{q}$, and 0 otherwise. The exponent $q$ can take different values, as commented in the introduction, with similar behaviour, although the results described here correspond to a dipole-dipole decay: that is $q=3$.

The parameter $\varepsilon$ is used to tune the strength of the coupling. The vectors $\boldsymbol{r}_{n}=\left(x_{n}, y_{n}\right)$, describe the positions of the oscillators in the two-dimensional space where the system is embedded. We normalize the distances between them, so that $\left|\boldsymbol{r}_{n}-\boldsymbol{r}_{n+1}\right|=1$, i.e. we neglect the stretching of the chain. The nearest-neighbour interaction can be recovered for $q=\infty$. The shape of the chain is described as a parabola, i.e. $y_{n}=\kappa x_{n}^{2} / 2$, with curvature $\kappa$. It can be an approximation of a more general shape in the neighbourhood of the bending point.

Note that the coupling coefficients, $J_{n m}$, do not depend on time, as the shape of the system is constant in time, although dependent on the curvature as a parameter.

This leaves only two parameters in our system: $\varepsilon$, the coupling parameter, which represents the ratio of the coupling energy with respect to the on-site energy, and the curvature of the system, $\kappa$, which describes the shape of the system.

The dynamical equations become

$$
\ddot{u}_{n}+\omega_{0}^{2} u_{n}-u_{n}^{3}+\varepsilon \sum_{m=1}^{N} J_{n m}\left(u_{n}-u_{m}\right)=0 \quad n=1, \ldots, N .
$$

\section{Methods}

We look for breathers: that is, localized, time-reversible, periodic solutions of (2). The procedure consists of expressing the functions $u_{n}$ as truncated cosine Fourier series of specified frequency $\omega_{\mathrm{b}}$, that is $u_{n}=z_{0, n}+\sum_{k=1}^{k_{m}} 2 z_{k, n} \cos \left(k \omega_{\mathrm{b}} t\right)$, and collecting together the coefficients corresponding to different $k$. Thus, we obtain a system of algebraic equations on $z_{k, n}$. Starting from the anticontinuous limit, i.e. $\varepsilon=0$, where we have solutions for the isolated variables, we can continue them using the Newton method up to some unspecified value of $\varepsilon$. The time reversibility condition allows us to eliminate the trivial time shift degeneracy. The theorem of 
existence can be found in the basic mathematical paper [1], and a thorough description of the numerical methods can be found in $[2,3,5]$. The linear stability is checked by studying the eigenvalues of the monodromy [4,25], i.e. the matrix $M_{0}$, given by

$$
\left[\left\{\xi_{n}(T)\right\},\left\{\dot{\xi}_{n}(T)\right\}\right]=M_{0}\left[\left\{\xi_{n}(0)\right\},\left\{\dot{\xi}_{n}(0)\right\}\right]
$$

where $T=2 \pi / \omega_{\mathrm{b}}$ is the period of the breather, and $\left[\left\{\xi_{n}(t)\right\},\left\{\dot{\xi}_{n}(t)\right\}\right]$ is the column matrix of positions and velocities of the solutions of the linearization of (2): that is, the result of the numerical integration (a symplectic integrator is highly recommended to get accurate results [27]) of

$$
(\mathcal{N}(u(t), \varepsilon) \cdot \xi)_{n} \equiv \ddot{\xi}_{n}+\omega_{0}^{2} \xi_{n}+s \Phi^{\prime \prime}\left(u_{n}\right) \xi_{n}+\varepsilon \sum_{m=1}^{N} J_{n m}\left(\xi_{n}-\xi_{m}\right)=0 \quad n=1, \ldots, N
$$

Here $\xi=\left\{\xi_{n}(t)\right\}$ represent the perturbations of the breather solution, and are not restricted to time reversible functions. The operator $\mathcal{N}(u(t), \varepsilon) \equiv\left\{\mathcal{N}_{n}(u(t), \varepsilon)\right\}$ is called the Newton operator. Therefore, the solutions of (4) can be described as the eigenvectors of $\mathcal{N}$ corresponding to the eigenvalue $E=0$.

As the linearized system is real and Hamiltonian, the monodromy is also a real and symplectic operator. This implies that if $\lambda$ is an eigenvalue of $M_{0}$, so are $\lambda^{*}, 1 / \lambda$ and $1 / \lambda^{*}$. Stability means that all the eigenvalues have modulus 1 . The fact that the derivative of the breather $\left\{\dot{u}_{n}(t)\right\}$ is a solution of (4) means that there is always a double eigenvalue at $1+0 \mathrm{i}$.

\section{Linear modes}

One of the first effects to take into account when the curvature is increased is the effect on normal modes. For a given $\kappa$ the linear mode frequencies form a band whose width increases with the coupling parameter $\varepsilon$, the phonon band. At the top of the band these modes are localized and at the bottom they are spread all over the system. The mode of lowest frequency, i.e. $\omega=\omega_{0}$, consists of all the oscillators vibrating in phase.

On the one hand, the curvature causes the band to become somewhat wider, thus apparently limiting the range of existence of the breather and its stability. In figure 2 can be seen the splitting of the phonon band with increasing coupling for $\kappa=0$ and 10 .

On the other hand, the curvature induces a larger difference of coupling constant between the oscillators, increasing the inhomogeneity of the system and, therefore, increasing Anderson localization. The top of the band modes are highly localized and can reinforce the breathers in the same site, provided they have similar shape, or could destroy them in the opposite case. This can be seen in figure 3 by comparing the shape of the modes with highest frequency for curvatures $\kappa=0$ and 20 for the same value of the coupling parameter $\varepsilon=0.3$.

\section{Stability of the central particle}

We can continue the one-site breathers located in any of the sites of the system. At zero coupling, all of them exist with any frequency $\omega_{\mathrm{b}}$ above $\omega_{0}$, if the potential is hard, and below $\omega_{0}$ if the potential is soft. They can be continued and are stable up to some unspecified value of the coupling $\varepsilon$ as long as the frequencies of the breather and its harmonics do not coincide with $\omega_{0}$ and the potential is nonlinear at that value of $\omega_{\mathrm{b}}$, i.e. $\partial \omega / \partial I \neq 0$. With increasing coupling the phonon band will split and eventually reach one of the harmonics, but, frequently, another localized mode separates from the band and produces the instability. The stability is analysed 

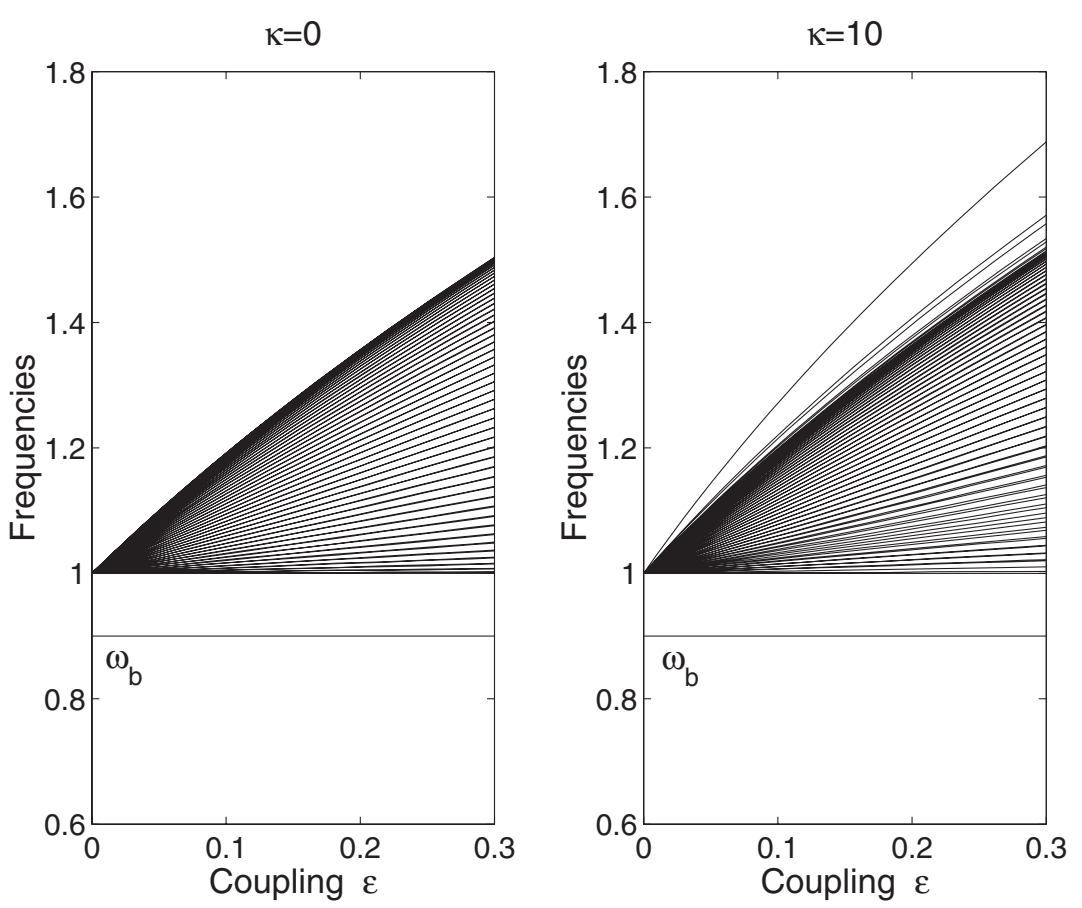

Figure 2. Splitting of the phonon band with increasing coupling for $\kappa=0$ and 10. Also shown is the breather frequency for a soft, on-site potential.
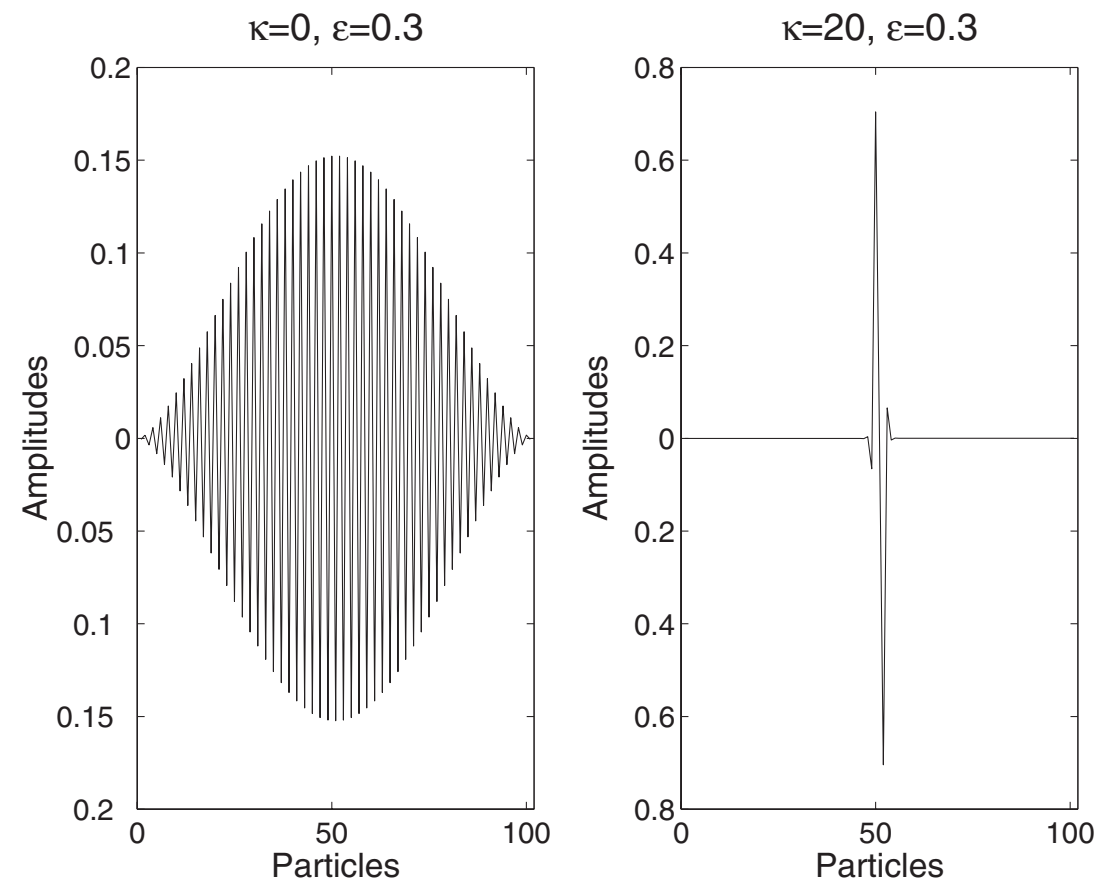

Figure 3. Linear modes of highest frequency $\kappa=0$ and 20 and coupling parameter $\varepsilon=0.3$. 

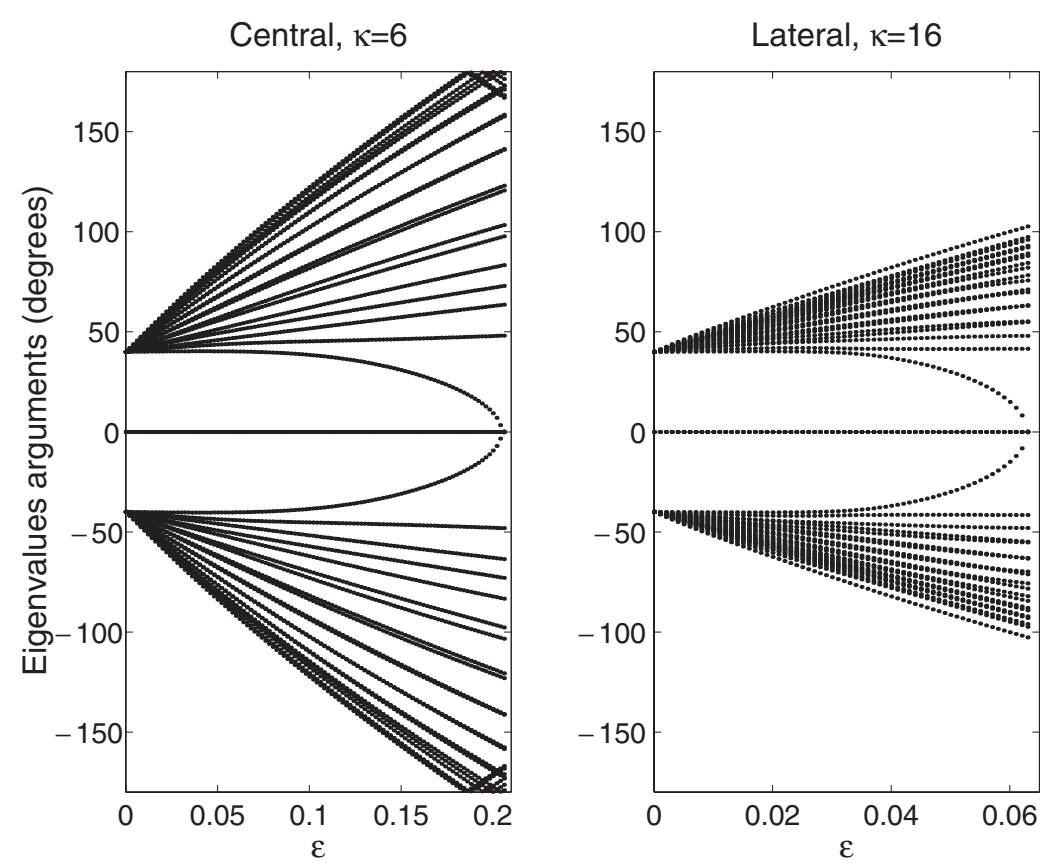

Figure 4. Two examples of the evolution of the monodromy eigenvalues with the coupling parameter. Left, for the breather on the bending point and $\kappa=6$. The eigenvalues cross at -1 but without producing any instability. Right, for the lateral breather, with $\kappa=16$. In fact, they are identical, as it is a pair of localized eigenvalues with frequency below the phonon band which reach +1 and abandon the unit circle, producing the instability.

observing the evolution of the eigenvalues of the monodromy. As commented above, they are all of modulus unity as long as the system is stable. The instability can be produced only in three different forms [4]: (a) a couple of conjugate eigenvalues reach 1 and leave the unit circle at the real line (harmonic resonance); (b) a couple of conjugate eigenvalues reach -1 and leave the unit circle at the real line (sub-harmonic resonance); (c) two pairs of conjugate eigenvalues collide at two points of the unit circle and leave it (Krein collision).

Figure 4 (left) shows the evolution of the arguments of the monodromy eigenvalues for the central particle with an intermediate curvature $\kappa=6$ until a harmonic resonance occurs. Note that it is a localized mode which separates from the phonon band and produces the instability. Similar pictures are obtained for different curvatures, increasing the splitting of the phonon band with increasing $\kappa$. Above a certain value they reach -1 , and thereafter many crossings of pairs of eigenvalues take place, although without any of them leaving the unit circle: that is, without affecting the breather stability. This can be interpreted in the framework of the band theory as that the eigenvalues belong to different bands of the equation $\mathcal{N}(u(t)) \cdot \xi(t)=E \xi(t)$ [4]. In figure 4 (right) is shown the evolution of the arguments of the monodromy eigenvalues for the lateral breather. The main difference is that the evolution of the eigenvalue that goes to produce the instability at +1 , i.e. $\theta=0$, is much faster. It reaches +1 for smaller values of the coupling parameter $\varepsilon$, when the phonon spectrum has not yet reached -1 and without any crossing of eigenvalues at this point. This is the main difference that appears in the evolution of the monodromy eigenvalues while considering the three different kinds of breathers and different values of the curvature. However, it turns out that the crossings in this model have no relevance, as the instability is always produced by a 


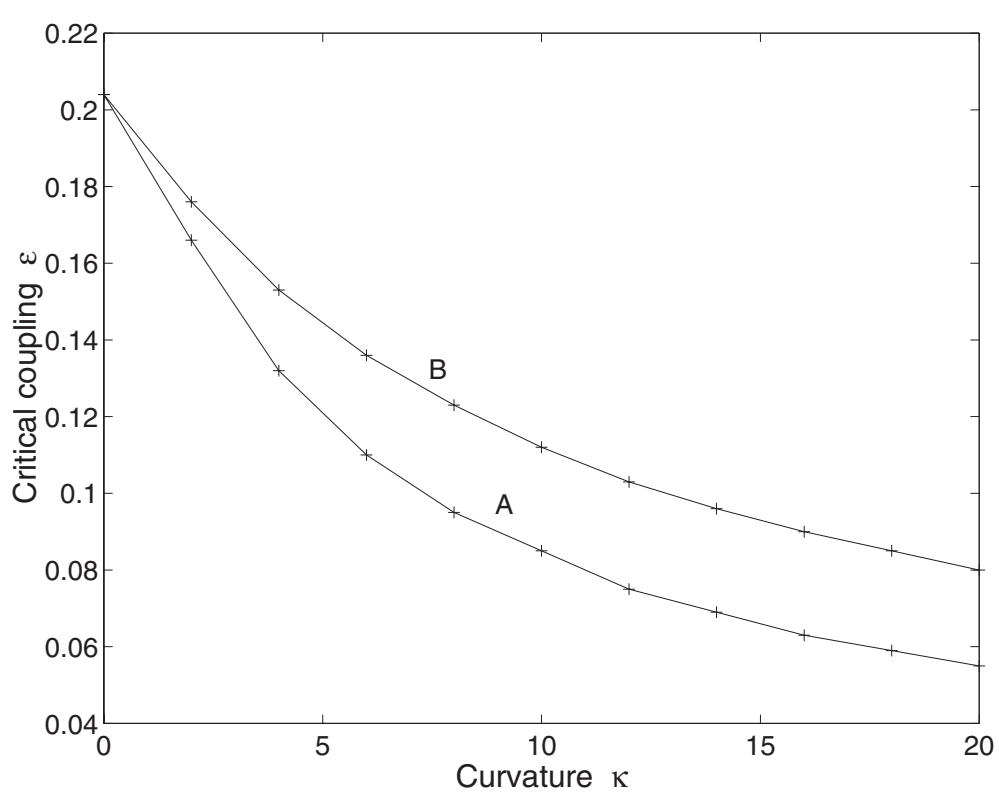

Figure 5. Bifurcation curves from linear stability to linear instability for breathers at the first (curve A) and second (curve B) neighbours of the one on the bending point. The regions of stability are the areas below these curves. The calculated points are represented by crosses.

localized eigenvalue with frequency smaller that the phonon band and leaving the unit circle at +1 .

Up to even the unrealistic values such as $\kappa=20$ that we have studied, the instability is produced in the same way and approximately for the same value of the coupling parameter. This means that the one-site breather localized at the bending point is highly stable, and not too much affected by the bending of the chain. Moreover, the eigenvector that produces the instability is centred on the bending-point particle. Therefore, it will not tend to delocalize the breather.

\section{Stability of the lateral particle}

We denote the particle next to the particle localized at the bending point as the lateral particle. The analysis of the stability of the breather located on this site gives a different picture than the one corresponding to the central particle. The evolution of the monodromy eigenvalues looks very similar to the breather at the bending point, as shown in figure 4 , but with two important differences. (a) The value of the coupling where the instability is produced, $\varepsilon_{\mathrm{c}}$, becomes smaller when the curvature $\kappa$ increases (while being approximately constant for the central breather). The space of parameters $(\kappa, \varepsilon)$ splits into two regions, divided by the bifurcation locus, given by the curve $\varepsilon_{\mathrm{c}}(\kappa)$. The stable region is the area below this curve, and the unstable region is the area above it. The curve $\varepsilon_{\mathrm{c}}(\kappa)$ is shown in figure 5 (also for the second breather). (b) With $\kappa$ large enough, the breather and the instability eigenvector extend to the central particle and to the symmetric one. This means that they will be affected by the instability. The profile of the instability eigenvector for some values of the parameters can be seen in figure 6 . The eigenvectors corresponding to non-real eigenvalues can be cast [26] (by multiplying by a complex scalar) into the form $\Omega=\left[\delta x_{n}, \mathrm{i} \delta v_{n}\right]$, while the eigenvectors corresponding to real eigenvalues are real. This implies that when $\Omega$ approaches the bifurcation point +1 , either its 


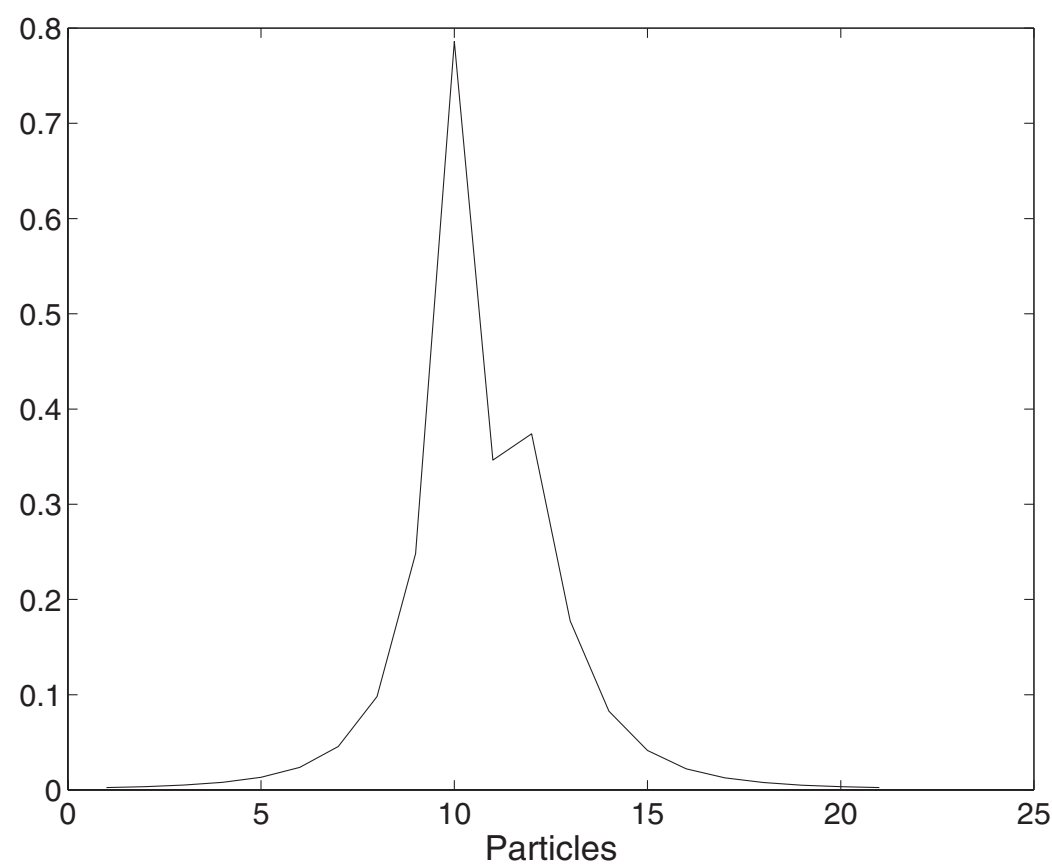

Figure 6. Components on the second half (the velocities part) of the instability eigenvector for $\kappa=10$ and critical coupling $\varepsilon=0.085$. The first half (the positions part) or the components are zero.

real part or its imaginary part tends to zero, so as it can be cast into a real vector. Therefore, at the bifurcation point, the eigenvector will be either $\left[\delta x_{n}, 0\right]$ or $\left[0, \delta v_{n}\right]$. The last one corresponds to our case, which is why only the second part is represented in figure 6 .

This analysis suggests that a change of position can be experienced by the lateral breather changing its position to the bending point. This can be tested by numerical integration of (2), with initial conditions corresponding to the lateral breather at the critical point. The simulation spectacularly confirms this suggestion. Although the time consuming calculation of eigenvalues has been done with a fairly small system of 21 particles, the simulation has been done with a larger system of 101 particles to eliminate the effect of reflection of the radiation emitted during the switching of position. This can be observed in figure 7 (left).

\section{Stability of the second neighbour}

Similar results are obtained with the second neighbour of the central particle. If this is continued while increasing the coupling, it eventually becomes unstable, due to some mode which detaches from the phonon band. The values of $\varepsilon_{\mathrm{c}}$ where the instability takes place are smaller than at the central site but higher than that corresponding to the lateral one, as can be seen in figure 5. Simulations performed within the instability parameters show that, frequently, the second-neighbour breather jumps to the first-neighbour site and almost immediately to the central site. A great deal of radiation is emitted, which means that the solution after the switching is not an exact one. The result, a nonlinear symmetric mode centred in the central particle, is not an exact breather, as it oscillates with several frequencies, but it remains stable and localized. The change of position can be seen in the right-hand side of figure 7 . 

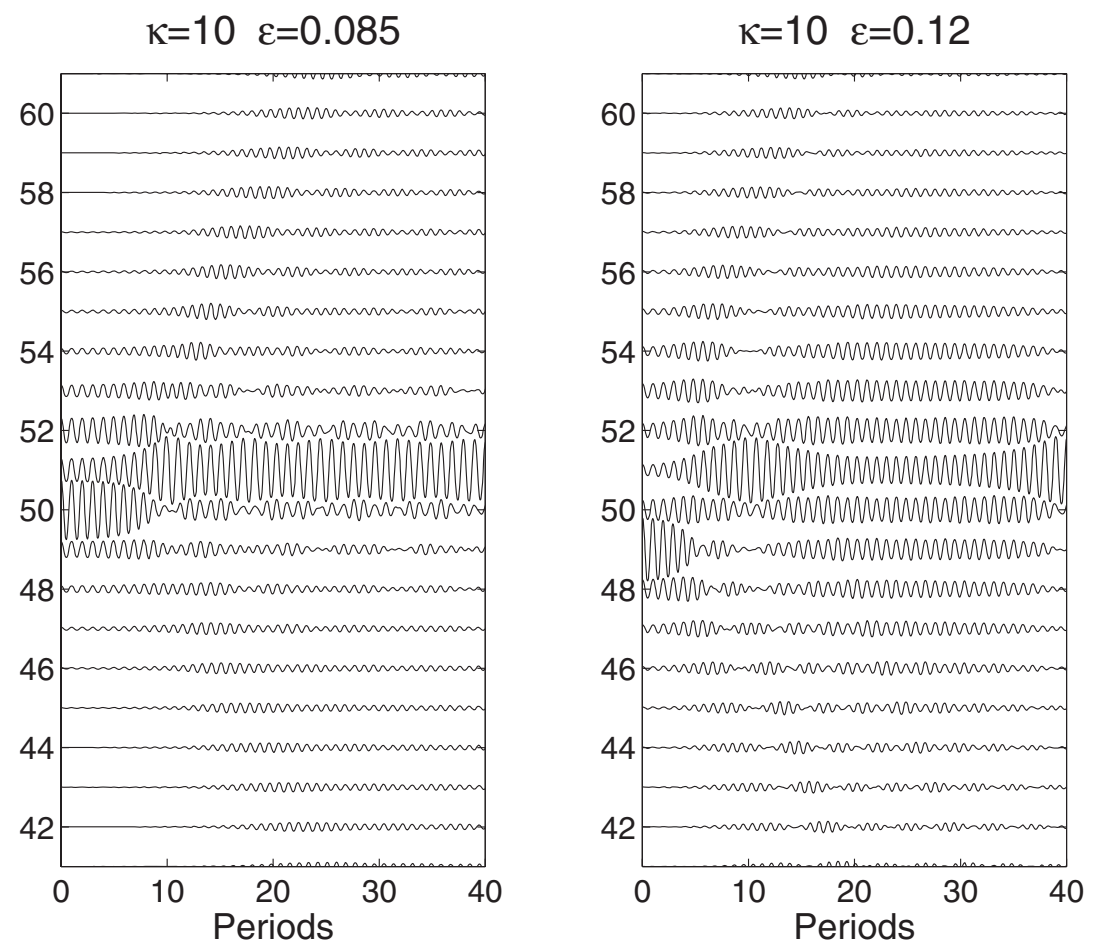

Figure 7. Switching of site of the lateral breather to the central site (left), and of the secondneighbour breather (right) in a model of 101 particles, with the values of the parameters shown. Particle 51 is the one on the bending point.

\section{Discussion}

The fact that a breather changes its position a site or two sites in a chain of oscillators can be seen as a special case of moving breather, i.e. a breather that moves one or two steps.

The theory of moving breathers is by no means so well developed as in the static case. Nevertheless, there is a systematic method to obtain them, which is very often successful [26]. A basic fact is that a stable breather cannot move or be made mobile by a small perturbation, because that would be in contradiction with its stability. Therefore, the mentioned method consists of obtaining a breather very close to an instability and perturbing it in the direction of an instability eigenvector.

The situation we are describing is quite similar. By changing the curvature of the chain (or the coupling), we obtain an unstable breather, with a pair of eigenvalues of the monodromy matrix reaching +1 and abandoning the unit circle. The breather moves, but through an inhomogeneous medium, due to the different coupling constants caused by the curvature. At the next position the conditions correspond again to a stable breather and it stops and adapts its shape to its new environment with the emission of some phonons. Or, as in the case of the second breather, the conditions can be so as it can move another step. The site at the bending point is the natural end of this short trip, as there the breather is far away from the instability. 


\section{Conclusions}

We address the problem of the existence and instability of breathers in a Klein-Gordon system of oscillators, with harmonic, long-range interaction coupling and coupling constants dependent on the distance between oscillators, taking into account the curvature of the chain.

We look for breathers and study their stability at the bending point and in its neighbourhood. Different behaviour is observed at these sites: the central breather is extremely stable and independent of the curvature, while the stability of the breathers located in the immediate neighbourhood of the bending point decreases rapidly with increasing curvature.

Numerical simulation show that breathers in the lateral particles, for values of the coupling and curvature where they are unstable and the central one is stable, jump, after a certain time, to the central site. Similarly, the value of the critical coupling diminishes with the curvature for the second-neighbour breather, which, frequently, jumps to the first neighbour and to the central particle. These results show a much higher probability of localization of energy in the bending points.

\section{Acknowledgments}

This work has been supported by the European Union under the RTN project, LOCNET, HPRN-CT-1999-00163. JFRA, SFM and YBG acknowledge Informatics and Mathematical Modelling for hospitality.

\section{References}

[1] MacKay R S and Aubry S 1994 Proof of existence of breathers for time-reversible or Hamiltonian networks of weakly coupled oscillators Nonlinearity 7 1623-43

[2] Marín J L and Aubry S 1995 Breathers in nonlinear lattices: numerical methods based on the anti-integrability concept Nonlinear Klein-Gordon and Schrödinger Systems: Theory and Applications ed L Vázquez, L Streit and V M Pérez-García (Singapore: World Scientific) pp 317-23

[3] Marín J L and Aubry S 1996 Breathers in nonlinear lattices: numerical calculation from the anticontinuous limit Nonlinearity 9 1501-28

[4] Aubry S 1997 Breathers in nonlinear lattices: existence, linear stability and quantization Physica D 103 201-50

[5] Marín J L, Aubry S and Floría L M 1998 Intrinsic localized modes: discrete breathers, existence and linear stability Physica D 113 283-92

[6] Flach S and Willis C R 1998 Discrete breathers Phys. Rep. 295 181-264

[7] Swanson B I, Brozik J A, Love S P, Strouse G F, Shreve A P, Bishop A R, Wang W Z and Salkola M I 1999 Observation of intrinsically localized modes in a discrete low-dimensional material Phys. Rev. Lett. 82 3288-91

[8] Russell F M, Zolotaryuk Y, Eilbeck J C and Dauxois T 1997 Moving breathers in a chain of magnetic pendulums Phys. Rev. B 55 6304-8

[9] Schwarz U T, English L Q and Sievers A J 1999 Experimental generation and observation of intrinsic localized spin wave modes in an antiferromagnet Phys. Rev. Lett. $83223-6$

[10] Trias E, Mazo J J and Orlando T P 2000 Discrete breathers in nonlinear lattices: experimental detection in a Josephson array Phys. Rev. Lett. 84 741-4

[11] Binder P, Abraimov D, Ustinov A V, Flach S and Zolotaryuk Y 2000 Observation of breathers in Josephson ladders Phys. Rev. Lett. 84 745-8

[12] Binder P, Abraimov D and Ustinov A V 2000 Diversity of discrete breathers observed in a Josephson ladder Phys. Rev. E 62 2858-62

[13] Sepulchre J A and MacKay R S 1998 Discrete breathers in disordered media Physica D 113 342-5

[14] Archilla J F R, MacKay R S and Marín J L 1999 Discrete breathers and Anderson modes: two faces of the same phenomenon? Physica D 134 406-18

[15] Kopidakis G and Aubry S 1999 Intraband discrete breathers in disordered nonlinear systems i: delocalization Physica D 130 155-86 
[16] Ting J J L and Peyrard M 1996 Effective breather trapping mechanism for DNA transcription Phys. Rev. E 53 1011-20

[17] Forinash K, Cretegny T and Peyrard M 1997 Local modes and localization in a multicomponent nonlinear lattice Phys. Rev. E 55 4740-56

[18] Gaididei Yu B, Mingaleev S F, Christiansen P L and Rasmussen K Ø 1997 Effects of nonlocal dispersive interactions on self-trapping excitations Phys. Rev. E 55 6141-50

[19] Mingaleev S F, Christiansen P L, Gaididei Yu B, Johansson M and Rasmussen K Ø 1999 Models for energy and charge transport and storage in biomolecules J. Biol. Phys. 25 41-63

[20] Christiansen P L, Johanson M, Gaididei Yu B and Rasmussen K Ø 1998 Solitary excitations in discrete twodimensional nonlinear Schrödinger models with dispersive dipole-dipole interactions Phys. Rev. B 5711303 18

[21] Gaididei Y B, Mingaleev S F and Christiansen P L 2000 Curvature-induced symmetry breaking in nonlinear Schrödinger models Phys. Rev. E 62 R53-6

[22] Christiansen P L, Gaididei Y B and Mingaleev S F 2001 Effects of finite curvature on soliton dynamics in a chain of nonlinear oscillators J. Phys.: Condens. Matter $201181-92$

[23] Flach S 1998 Breathers on lattices with long range interaction Phys. Rev. E 58 R4116-9

[24] Baesens C and Mackay R S 1999 Algebraic localization of linear response in networks with algebraically decaying interaction, and application to discrete breathers in dipole-dipole systems Helv. Phys. Acta 72 23-3

[25] MacKay R S and Sepulchre J A 1998 Stability of discrete breathers Physica D 119 148-62

[26] Aubry S and Cretegny T 1998 Mobility and reactivity of discrete breathers Physica D $11934-46$

[27] Sanz-Serna J M and Calvo M P 1994 Numerical Hamiltonian Problems (London: Chapman and Hall) 